\section{RUPTURE OF THE UTERUS DURING PREGNANCY}

BY

\author{
JAMES RIDDELL, M.D., F.R.C.S.Ed., M.C.O.G. \\ Honorary Gynaecological Surgeon, Prince of Wales's \\ Hospital, Plymouth \\ AND
}

JOHN SCHOLEFIELD, M.B., Ch.B., F.R.C.S.

Resident Surgical Officer, Prince of Wales's Hospitat, Plymouth

The case of rupture of the uterus during pregnancy which is described in detail and discussed in this paper was that of a primigravida, aged 20, admitted to the Prince of Wales`s Hospital, Plymouth.

\section{Clinical History}

The patient was thirty-two weeks pregnant, and had been attending an ante-natal clinic and had enjoyed health during the whole of her pregnancy. There was no history of any pelvic pain even during the early weeks of pregnancy. On the morning of admission to hospital she sat up in bed and was seized with severe abdominal pain. During the course of the morning she sent for a midwife, who found her walking about her room complaining of a pain in the lower part of the abdomen on the right side. The midwife put the patient to bed and sent for the family doctor. He sent the patient to hospital, having diagnosed acute appendicitis. She was admitted to hospital shortly after midday, and was observed by one of us (J. S.).

On admission the patient looked pale and toxic. There was not any restlessness, but her respirations were rather rapid and there was some dyspnoea. The pain was referred to the right hypochondrium and was of a colicky nature. There was some tenderness in the right hypochondrium, but not any rigidity, and pressure here resulted in a series of short, rapid respirations. The uterus appeared normal for a thirty-two-week pregnancy, and a living child in the left occipito-anterior position was diagnosed.

The urine was carefully examined to exclude a pyelitis and a physician's opinion obtained to exclude an acute diaphragmatic pleurisy. The tenderness appeared to be too high for appendicitis and the history did not support such a diagnosis. A provisional diagnosis of an acute cholecystitis complicating pregnancy was made, and the patient was placed in the Fowler position and put on fluids for observation. During the next few hours the pain did not show any signs of abating and the tenderness had increased. A further opinion was sought.

\section{Further Examination of the Case}

The patient was referred to me (J. R.) at 10 p.m. on the evening of her admission to hospital. She was complaining of severe pain under the costal margins and under the shoulderblades and was in great distress, particularly with dyspnoea. There was not any pain referred to the shoulders.

The outline of the uterus was regular and was clearly defined. The uterus was enlarged to the size of a thirty-twoweek pregnancy. The foetal heart sounds were heard. Above the level of the uterus the abdominal wall was flaccid, but there was pronounced tenderness under the costal margins. There was slight bleeding from the vagina. The external os was closed. The patient was not in labour. Examination of the chest did not disclose anything abnormal. The urine was free from pus cells and $B$. coli. The temperature was $98.8^{\circ}$, pulse 120 , respirations 28 .
I was not able to make a diagnosis, though I considered that an acute abdominal emergency might be in progress, possibly an internal haemorrhage. I was not able to satisfy myself that there was sufficient evidence to warrant opening the abdomen that night. I desired to wait and to make further observation. An hourly pulse chart was arranged for, and a request was made that $I$ should be called if the pulse rate increased appreciably. A third of a grain of omnopon was administered hypodermically. The patient slept, and the pulse rate fell to 90 by 6 a.m.

I saw the patient next day shortly before 1 p.m., when I found that the clinical picture had changed. The patient was sleeping, but her face had a more distressed expression; she looked pale, with shallower, rather quicker, and slightly more embarrassed respiration.

The abdominal wall was rigid and tense, the uterine outline was not manifest any longer, there was a more diffuse swelling of the abdomen due to some distension. Liver dullness was absent. I made a diagnosis of internal haemorrhage. Rupture of the uterus was discussed, but there was not sufficient evidence to support that diagnosis with confidence. There was only sufficient evidence to warrant a diagnosis of internal haemorrhage.

\section{Operation}

The patient was removed to the operating theatre. I opened the peritoneal cavity through a left paramedian incision, partly above the umbilicus and partly below, in order that I might have liberty to enlarge it upwards or downwards, depending upon what was discovered to be the source of the bleeding.

On opening the peritoneal cavity a bag of membranes was seen. Rupture of the uterus was now obvious. The incision was extended downwards. The uterus was felt contracted in the bottom of the pelvis. The foetus (dead), membranes, and placenta were removed. The uterus was lifted up. At the right cornu a four-inch rent extended down the right side of the uterus. The uterus was elongated and marrow-shaped.

At the site of the rent the wall of the uterus was thin. Bleeding was not in progress, though there was a considerable quantity of blood in the peritoneal cavity. I did not waste time exploring the inside of the uterus. The right tube was not continuous with the uterine wall. It looked natural and healthy, except that it ended proximally in a healed stump, as though it had been amputated. The centre of the rent in the uterus corresponded with the point at which the tube would normally have become continuous with the uterus. The right ovary was calcified. The left tube and ovary were natural.

The rent in the uterus was sutured, most of the blood was removed from the peritoneal cavity, drainage was provided, and the patient was returned to bed as quickly as possible. She recovered quickly, and went home on the fifteenth day after operation.

\section{Remarks by Mr. James Riddell}

In the case of rupture of the uterus during pregnancy which I described in 1926 there had been previous damage to the uterus following miscarriages. Hysterectomy was employed and the patient recovered. In the case now under review there had not been any operation upon the uterus. There was not any history of injury or violence of any kind.

Munro Kerr has described "angular" pregnancy, and he states that atypical examples may be encountered, partly " inferstitial,". partly " angular." It is suggested that this case is an example of a pregnancy partly "interstitial," partly "angular." Munro Kerr quotes Kupferberg, who described a case in which the body of the child was in the tube and the head in the uterus. The "interstitial" element would account for the erosion of the tube by the 
trophoblast and its gradual amputation from the body of the uterus. It is not possible for a developmental defect to account for the want of continuity between the tube and the uterus. The tube was properly developed, and was not in any way under-developed. The uterus was properly and naturally developed. It is possible that after the erosion of the tube the ovum may have moved a little more downwards and embedded itself more comfortably in the uterus.

The ovum developed not in substantia uteri but towards the uterine cavity, though almost wholly in it, and that constitutes the "angular" element which led to thinning out of the wall of the uterus at the right cornu. Eventually the wall of the uterus became so thin that the exertion of sitting up in bed was enough to rupture it. There was not any recognizable lateral distension of the uterus in the region of the uterine cornu. Obliquity of the uterus was not observed before the operation, and there was not any evidence of it at the operation. There was only thinning of the wall at the right cornu.

Lateral distension of the uterine cornu is one of the main features described by Munro Kerr. Therefore there must be grades of cases-if my explanation is correctsome without much iateral distension of the uterus. Most "angular" pregnancies produce great pain and lead to abortion. If there were not much sacculation at the cornu but only thinning of the uterine wall, and if the ovum found a more secure resting place, then abortion would not be likely to occur. This would explain why the patient did not abort.

If my explanation is not correct, then one must suppose that the ovum was properly embedded in the uterus from the start and that the amputated tube and the thinned-out cornu did not have any relation to it, but were entirely unconnected with it, being due to some pre-existing unexplained disease or injury.

The patient has promised to report if she becomes pregnant again in order that her progress may be watched.

\section{Summary}

1. The clinical history of a case of rupture of the uterus during pregnancy is reported. The patient recovered after suture of the rent.

2. It is suggested that the cause of the rupture was a pregnancy partly "interstitial," partly "angular."

\section{BibliogRAPHY}

Munro Kerr, J. M. (1937). Operative Obstetrics, p. 700; (1934) British Medical Journal, 1, 1113.

Riddell, J. (1926). J. Obstet. Gynaec. Brit. Emp., 33, 1.

The two Chambers of the Swedish Riksdag have passed a Bill for public dental care. The new Act provides for dental treatment for all infants and school children, as well as for adults, at a nominal charge, or free of charge in the case of families of small means. As regards children public dental treatment will be available to all between the ages of 3 and 15 years. The annual fee is $5 \mathrm{kr}$. for the first child, and $3 \mathrm{kr}$. and $2 \mathrm{kr}$. for the next two children, of a family registered at one and the same time for receiving State dental care. If there are more than three children in a family the rest will be treated free. As regards adults, treatment is to be given at cost price, or at a reduced fee or gratis when warranted. The Act provides for a division of Sweden into dental districts, with whole-time dentists and attendants for each. The cost of organizing and maintaining the service will be divided between the State and the county councils or municipalities.

\section{Clinical Memoranda}

\section{Concurrent Puerperal Anaerobic Streptococcal and Staphylococcal Septicaemia with Recovery}

We feel that points worthy of notice in this case are, first, the unusual combination of organisms in the blood stream ; secondly, the response to staphylococcal antitoxin in a patient who was gravely ill ; and, thirdly, the presence of septicaemia after a considerable amount (66 grammes) of sulphanilamide had been given. The response to the staphylococcal antitoxin suggests that the presence of the anaerobic streptococci in this case was of minor importance.

Mrs. M., aged 32 years, was delivered of her second child on February 15, 1938. The first and second stages of labour were normal, but there was delay in the delivery of the placenta, which was removed manually under general anaesthesia. On the following day the patient complained of intense headache, shivering, and insomnia. She vomited several times. In the evening her temperature rose to $101^{\circ} \mathrm{F}$.

She was admitted to hospital on February 17 . Her temperature was $98^{\circ} \mathrm{F}$., pulse 108 , and respirations 24 . She was pale and appeared ill. The fundus of the uterus was palpable two inches below the umbilicus and was not tender. Vaginal discharge was scanty and haemorrhagic. Examination of other organs showed no abnormality. Sulphanilamide 4.5 grammes was given daily by mouth, and $1 \mathrm{c.cm}$. pituitrin hypodermically twice a day.

The day after admission she passed a piece of membrane per vaginam. Next morning her temperature rose to $101.2^{\circ} \mathrm{F}$. and her pulse rate to 120 per minute. Her condition became steadily worse, and on February 24 the temperature was $103.2^{\circ} \mathrm{F}$. and she had a slight rigor. On the following day $20 \mathrm{c.cm}$. scarlatinal antitoxin were given intramuscularly and $60 \mathrm{c.cm}$. intravenously on the 26th; after this her temperature fell to normal for the first time for seven days, but rose again next day and continued to swing between a maximum of $104.2^{\circ} \mathrm{F}$. and a minimum of $99^{\circ} \mathrm{F}$. for the next three weeks.

On March 3 a blood count showed: Red cells 2,164,000 per c.mm. White cells 3,600 per c.mm. (polymorphs 64 per cent., lymphocytes 32 per cent., monocytes 3 per cent., eosinophils 1 per cent.). Haemoglobin 30 per cent. Colour index 0.7. Reticulocytes 4.5 per cent. There was marked anisocytosis and some poikilocytosis of erythrocytes.

Blood culture on March 4 gave a growth of non-pigmented staphylococci which were thought to be due to contamination of the culture. Her clinical appearance now became typically that of septicaemia, and on March 6 she was given a transfusion of $15 \mathrm{oz}$. of her husband's blood; this was followed by some temporary improvement. On the 9th the blood culture was repeated and gave a similar growth of staphylococci, which were haemolytic and showed slight pigmentation of the aureus type at the end of forty-eight hours' incubation. An anaerobic culture was made simultaneously, and this showed the presence of anaerobic streptococci as well.

It was now decided to give staphylococcus antitoxin. As she had had serum a fortnight previously she was given two small desensitizing doses of the antitoxin subcutaneously at an interval of three hours, and later the same day (March 12) 10,000 units intramuscularly. This latter dose was repeated on the following day. By this time her condition was very poor: temperature $104^{\circ} \mathrm{F}$., pulse rapid, skin yellow, and mucous membranes pale; there was a systolic bruit at the apex and she was incontinent of urine and faeces. She had also developed a massive generalized oedema involving all limbs, face, and back, which may have been due to the serum. 